\title{
BMJ Open Systematic review and meta-analysis of the incidence and prevalence and 30- year trend of inflammatory bowel diseases in Asia: a study protocol
}

\author{
Ali Reza Safarpour, ${ }^{1}$ Manoosh Mehrabi, ${ }^{2}$ Abbasali Keshtkar, ${ }^{3}$ Fardad Edjtehadi, \\ Kamran Bagheri Lankarani ${ }^{4}$
}

To cite: Safarpour AR, Mehrabi M, Keshtkar A, et al. Systematic review and meta-analysis of the incidence and prevalence and 30 -year trend of inflammatory bowel diseases in Asia: a study protocol. BMJ Open 2019;9:e031854. doi:10.1136/ bmjopen-2019-031854

- Prepublication history and additional material for this paper are available online. To view these files, please visit the journal online (http://dx.doi org/10.1136bmjopen-2019031854).

Received 02 June 2019 Revised 08 0ctober 2019 Accepted 14 October 2019

Check for updates

(C) Author(s) (or their employer(s)) 2019. Re-use permitted under CC BY-NC. No commercial re-use. See rights and permissions. Published by BMJ.

${ }^{1}$ Gastroenterohepatology Research Center, Shiraz University of Medical Sciences, Shiraz, Iran

${ }^{2}$ Department of E-Learning, Virtual school, Shiraz University of Medical Sciences, Shiraz, Iran ${ }^{3}$ Department of Health Sciences Education Development,

School of Public Health, Tehran University of Medical Sciences, Tehran, Iran

${ }^{4}$ Health Policy Research Center, Shiraz University of Medical

Sciences, Shiraz, Iran

Correspondence to

Dr Manoosh Mehrabi;

mehrabi.manoosh@gmail.com

\section{ABSTRACT}

Introduction Inflammatory bowel diseases, including ulcerative colitis (UC), Crohn's disease (CD) and inflammatory bowel disease type unclassified (IBDU), are debilitating conditions that are rapidly growing in developing countries. Given the absence of a comprehensive systematic review and meta-analysis containing a rigorous pooled estimate of incidence and prevalence of UC, CD and IBDU, this study was conducted to determine the incidence and prevalence of these conditions in Asia and their 30-year trend.

Methods and analysis Based on predefined criteria, electronic databases, including PubMed/MEDLINE, Scopus, WoS (Clarivate Analytics), Embase and Google Scholar, and some databases pertaining to Asian countries will be searched for population-based cross-sectional studies and the baseline data and final reports of population-based cohort studies involving paediatric and adult patients, with no language restrictions, from 1 January 1988 to 30 December 2018. Any disagreement in the stages of screening, selecting, quality assessment and data extraction between the two independent reviewers will be resolved by consensus, and if the disagreement is not resolved, a third expert opinion will be sought. The combination method will be used based on methodological similarities in the included studies by the Fixed Effect Model or the Random Effect Model. Forest plots will be plotted for all the studies to show the separated and pooled incidence and prevalence and their corresponding 95\% Cls. The Q-statistic test and $\mathrm{I}^{2}$ statistic will be used to assess statistical heterogeneity. Funnel plots will be used to assess potential reporting bias and nonsignificant study effect. Begg's and Egger's tests will also be performed, and significant results $(p>0.1)$ shall suggest a publication bias, in which case the 'trim and fill' method will be used. The time trends for UC, CD and IBDU will be calculated using a cumulative meta-analysis.

Ethics and dissemination Since this review will use previously published studies, it will not require the consent of an Ethics Committee. The results will be prepared and disseminated through a peer-reviewed journal and will be presented in relevant conferences.

PROSPERO registration number CRD42019131477.

\section{BACKGROUND}

Inflammatory bowel diseases (IBDs) include three chronic, non-curable and idiopathic
Strengths and limitations of this study

- This study will provide evidence on the Asian prevalence and incidence of ulcerative colitis, Crohn's disease and inflammatory bowel disease type unclassified.

- This study will combine data and assess the value and causes of possible heterogeneity.

- This study uses an inclusive search based on thesaurus systems, including Emtree and MeSh, and carries out its search in large databases, such as SCOPUS, WOS, MEDLINE/PubMed, Embase, Google Scholar and ProQuest, with a long time range.

- Given the lack of strong population-based studies in most countries in Asia, this review may not show the actual population-based prevalence and incidence of the diseases under study.

- Methodological biases in the primary studies included may cause uncertainty in the results of the present study.

diseases, namely ulcerative colitis (UC), Crohn's disease (CD) and inflammatory bowel disease type unclassified (IBDU), ${ }^{12}$ which are developed as a result of genetic, ${ }^{3}$ environmental ${ }^{4}$ and immunologic ${ }^{5}$ factors.

Given the absence of a histological or serological gold standard for confirming the diagnosis of IBD and also the abundance of diseases that mimic the symptoms of this disease, IBD is diagnosed based on a series of clinical, endoscopic and histological findings. ${ }^{6}$ The two most commonly used criteria in IBD diagnosis include the Lennard-Jones criteria $^{7}$ and the Mendeloff criteria. ${ }^{8}$ The diagnostic criteria stated in two other references, including the international multicenter scoring system of the Organisation Mondiale de Gastroenterologie ${ }^{9}$ and the diagnosis criteria of the Japanese Research Society on IBD, ${ }^{10}$ are also acceptable in this study. 
A definition of IBD by either of these four criteria is acceptable in this study, and the International Classification of Diseases, 10th Revision (ICD-10) diagnostic codes, that is, K51.0-51.9 for UC and K50.0-50.9 for CD, are approved for the diagnosis of these diseases. IBDU will be defined according to the ICD-10 code: K52.3 and the diagnostic criteria revealed by Guindi et $a l^{11}$ and Ouyang et al. ${ }^{12}$ Moreover, the incidence rate of IBD is the number of new cases of the disease over a given period of time in a specific geographical area, and the prevalence of IBD is the number of living IBD patients over a given period of time in a specific geographical area.

The highest prevalence of IBD is still seen in northern industrial countries, such as North American and European countries. The prevalence of IBD appears to have stabilised in these countries at over $0.5 \%$ in the general population. ${ }^{13}$ The incidence of UC and CD is 24.3 and 29.3 per 100000 of the population in these countries. ${ }^{14}$ These patients impose great annual costs on the health system; for example, in 2004, an estimated US $\$ 6$ billion was spent on IBD patients in the USA. ${ }^{15}$ The annual costs incurred by these patients were $\$ \mathrm{C} 1.2$ billion in Canada and $€ 5$ billion in Europe. ${ }^{16} 17$

Although the prevalence and incidence of IBD were low in Asia before the past two to three decades (due to non-diagnosis or the small number of cases), the prevalence and incidence of these diseases were severely exacerbated in Asian countries after this time as a result of their industrialisation. ${ }^{18} 19$

Many studies conducted in Asia have shown great differences in the epidemiological indices of IBDs. A study conducted in 2013 reported the incidence of IBD as 0.54 to 3.44 per 100000 of the population..$^{20}$ In South Korea, the incidence rates of UC and CD were reported as 4.6 and 3.2 per 100000 of the population. ${ }^{20}$ From 1991 to 2005 in Japan, the prevalence of UC increased from 18.1 to 63.6, and the prevalence of $\mathrm{CD}$ increased from 5.9 to 21.1 per 100000 of the population. ${ }^{21} 22$ The incidence of IBD increased 10-fold in South Korea over two decades. ${ }^{23}$ Despite the stabilisation in its incidence in advanced countries, IBD appears to be rapidly growing in Asia. ${ }^{1}$

In general, the chronic nature of these diseases, their small mortality rate, their trend of progression, that is, remission and exacerbation over the course of the disease, and the comorbidity of dysplasia and colon or rectal cancer in many of the patients ${ }^{24}$ impose a heavy financial burden on the health system of countries in terms of both disease treatment and complications.

Examining the epidemiological indices of the prevalence and incidence of the disease and investigating the reasons for the reduction or increase in these indices over the years (given the rapid trend of industrialisation in Asian countries and the increasing environmental risk factors) can help health policymakers calculate the burden of IBD in Asia. ${ }^{18}$

To the researchers' knowledge, three systematic reviews have been conducted to date on the prevalence and incidence of IBD, but without data combining, making estimates, examining the reasons for heterogeneity or conducting a meta-analysis. A valuable research was recently carried out by $\mathrm{Ng}$ et al in $2018^{25}$ that assessed the global prevalence and incidence of IBD (last accessed 31 December 2016) by conducting a search in MEDLINE and Embase. The Asian part of the study examined population-based studies conducted in 19 countries of this continent. According to the findings, in East Asia, the highest and lowest incidence rates were 3.2 and 0.06 for $\mathrm{CD}$ and 4.6 and 0.42 for UC per 100000 person-years, and the highest and lowest prevalence rates were 18.6 and 1.05 for CD and 57.3 and 4.59 for UC per 100000 of the population; in Southeastern Asia, the highest and lowest incidence rates were 0.41 and 0.14 for $\mathrm{CD}$ and 0.68 and 0.15 for UC per 100000 person-years, and the highest and lowest prevalence rates were 2.17 and 2.17 for $\mathrm{CD}$ and 6.67 and 6.67 for UC per 100000 of the population; in Southern Asia, the highest and lowest incidence rates were 3.91 and 0.09 for $\mathrm{CD}$ and 6.02 and 0.69 for UC per 100000 person-years, and the highest and lowest prevalence rates were 1.2 and 1.2 for $\mathrm{CD}$ and 44.3 and 5.3 for UC per 100000 of the population; in Western Asia, the highest and lowest incidence rates were 8.4 and 0.94 for $\mathrm{CD}$ and 6.5 and 0.77 for UC per 100000 person-years, and the highest and lowest prevalence rates were 53.1 and 50.6 for $\mathrm{CD}$ and 106.2 and 4.9 per 100000 of the population. This study also investigated the temporal trend of the incidence of these diseases over the three examined decades.

Another systematic review conducted by Molodecky et al $^{13}$ (last accessed 2010) also performed a search in MEDLINE and Embase and investigated 13 countries in Asia and the Middle East. The results showed that the incidence rate of UC ranged from 0.11 in Singapore to 6.52 in Panjab, India, per 100000 person-years, and the prevalence of UC also ranged from 4.9 in Turkey to 168 in Kibbutz, Israel, per 100000 of the population. ${ }^{13}$ The incidence of CD ranged from 0.04 in Singapore to five in Kibbutz, Israel, per 100000 person-years, and its prevalence ranged from 0.88 in Japan to 67.9 in Kibbutz, Israel, per 100000 of the population. This study did not entail data combining, making estimates, examining the reasons for heterogeneity or conducting a meta-analysis.

Another systematic review, ${ }^{26}$ conducted by Prideaux et al (last accessed October 2011), examined studies conducted in only nine Asian countries through a search in Medline (EBSCO Host) and Cochrane. The highest incidence of UC was observed in India (six cases $/ 10^{5}$ person-years), and the highest incidence of $\mathrm{CD}$ was observed in South Korea (5.1 cases $/ 10^{5}$ person-years). The highest prevalence rates of $\mathrm{UC}$ and $\mathrm{CD}$ were observed in Asakura, Japan (63.6 cases $/ 10^{5}$ person-years and 21.2 cases $/ 10^{5}$ of the population, respectively).

Two other review articles were found on the epidemiological indices of IBD (prevalence, incidence and risk factors), including one article by Thia et al in $2008^{18}$ on preliminary studies in eight Asian countries and another 
by Cosnes et $\mathrm{al}^{14}$ on preliminary studies conducted in some countries (including three Asian countries).

The present systematic review will be a more comprehensive examination of the preliminary studies on the subject in question compared with its predecessors because of its prior registration in PROSPERO, review of all the relevant studies regardless of age group (including paediatrics or adults), data combining, assessment of the value and causes of possible heterogeneity, inclusive search based on the use of thesaurus systems (Emtree and MeSh), search in large databases (SCOPUS, WOS, MEDLINE/PubMed, Embase, Google Scholar and ProQuest) with a longer time range, use of regional databases (Indian Citation Index, Chinese Citation Index, Korean Citation Index and five other large Chinese biomedical bibliographic databases ${ }^{27}$ and IranMedex), use of grey literature (theses and conference papers and proceedings) and also use of experts' views and examination of key journals.

Based on the results of previous studies conducted on the prevalence and incidence of IBD, there is a northsouth gradient and an east-west gradient between Western countries $^{28}$ and Asian countries in this respect. ${ }^{19}$ Nevertheless, this issue has not been specifically investigated using the dose-response method based on geographical longitude and latitude. Moreover, since Westernisation and industrialisation appear to be risk factors for the increased incidence and prevalence of IBD, ${ }^{29}$ and since the per-capita income is one of the indicators of industrialisation, the dose-response method shall be used, if possible, to investigate the relationship of this phenomena with the increased prevalence and incidence of $\mathrm{CD}, \mathrm{UC}$ and IBDU in Asian countries.

The present systematic review and meta-analysis was conducted to provide clinical professionals and healthcare system policymakers in Asia (the largest and most populated continent in the world with 50 countries) with the latest information about the prevalence and incidence of $\mathrm{CD}, \mathrm{UC}$ and IBDU so that these groups can more effectively deal with the challenges of the growing trend of IBDs over the next decade.

\section{OBJECTIVES}

\section{Primary objective}

The primary objective of the present systematic review and meta-analysis is to estimate the prevalence and incidence of $\mathrm{CD}$, UC and IBDU in patients (of any age, including paediatrics or adults) in Asia. ${ }^{30}$ According to United Nations, Department of Economic and Social Affairs, Statistics Division, the Asian Continent consists of five geographical subdivisions, including Central Asia (Kazakhstan, Kyrgyzstan, Tajikistan, Turkmenistan and Uzbekistan), Eastern Asia (China, China; Hong Kong Special Administrative Region, China; Macao Special Administrative Region, Democratic People's Republic of Korea, Japan, Mongolia and Republic of Korea), Southeastern Asia (Brunei Darussalam, Cambodia, Indonesia,
Lao People's Democratic Republic, Malaysia, Myanmar, Philippines, Singapore, Thailand, Timor-Leste and Vietnam), Southern Asia (Afghanistan, Bangladesh, Bhutan, India, Iran (Islamic Republic of), Maldives, Nepal, Pakistan and Sri Lanka) and Western Asia (Armenia, Azerbaijan, Bahrain, Cyprus, Georgia, Iraq, Israel, Jordan, Kuwait, Lebanon, Oman, Qatar, Saudi Arabia, State of Palestine, Syrian Arab Republic, Turkey, United Arab Emirates and Yemen).

\section{Secondary objectives}

1. Estimating the prevalence and incidence of $\mathrm{CD}, \mathrm{UC}$ and IBDU in Asia by age group.

2. Estimating the prevalence and incidence of $\mathrm{CD}, \mathrm{UC}$ and IBDU in Asia by gender.

3. Estimating the prevalence and incidence of $\mathrm{CD}, \mathrm{UC}$ and IBDU by the five geographical regions of Asia, including Central Asia, East Asia, Southern Asia, Southeastern Asia and Western Asia.

4. Estimating the prevalence and incidence of $\mathrm{CD}, \mathrm{UC}$ and IBDU in Asia by the latitude of the study country.

5. Estimating the prevalence and incidence of $\mathrm{CD}, \mathrm{UC}$ and IBDU in Asia by the per-capita income of the study country.

6. Determining the temporal trend of the prevalence and incidence of $\mathrm{CD}, \mathrm{UC}$ and IBDU over the last three decades.

7. Assessing potential heterogeneity in the prevalence and incidence of CD, UC and IBDU in Asia and finding its potential causes.

\section{METHODS AND DESIGN}

The protocol for this systematic review and meta-analysis was prepared according to the Center for Reviews and Dissemination guidelines ${ }^{31}$ and will be reported according to the Meta-analysis of Observational Studies in Epidemiology guidelines. ${ }^{32}$ The selection process of the studies will be reported according to the Preferred Reporting Items for Systematic review and Meta-Analysis- Protocols (PRISMA-P) $2015 .^{33}$

\section{STUDY ELIGIBILITY CRITERIA Inclusion and exclusion criteria Types of studies}

This study shall select all the population-based observational studies that have correctly (based on an acceptable definition) estimated or presented data on the prevalence and incidence (or both) of UC, CD or IBDU (or all) in Asia with which these indices can be calculated. These observational studies will include populationbased cross-sectional studies for estimating the prevalence and prospective, population-based, cohort studies (final results) for estimating the incidence. The studies should contain the numerator and denominator of the prevalence and incidence estimation fraction (to obtain 
the SE of the incidence/prevalence); if not, these data will be gathered through correspondence with the author.

Review articles, case reports, hospital studies and case series will not be included in the present study. Moreover, prospective, population-based, cohort studies (baseline data) will be used as a special design to estimate the prevalence indicator.

In this study, population-based studies refer to studies conducted on a representative population of a geographical region that have used a random sampling method and have a fairly equal gender distribution (about $50 \%$ from each gender) and also an age distribution that is consistent with the age distribution in the target population (or at least one of the study age groups in the representative sample should be similar to the corresponding age group in the target population).

\section{Types of participants}

The present study will include all the preliminary studies conducted in Asian countries ${ }^{30}$ on patients (male, female or both and at any age) with CD, UC or IBDU. Studies conducted on different Asian ethnicities or races will be included, provided that they meet the other inclusion criteria of this systematic review.

All the studies conducted outside Asia on Asian immigrates or refugees as well as those conducted in Asia on immigrants from other countries (Asian or non-Asian) will be excluded.

\section{Disease (outcome)}

This study accepts definitions of IBD (including CD or UC) based on either the Lennard-Jones ${ }^{7}$ or Mendeloff ${ }^{8}$ criteria. IBDU is defined as clinical and endoscopic manifestations of inflammatory bowel disease affecting the colon without showing any evidence of small bowel involvement and with no definitive histological or other types of evidence in favour of $\mathrm{CD}$ or UC. ${ }^{34}$ Moreover, the ICD-10 diagnostic codes (UC: K51.0-51.9, CD: K50.050.9 and IBDU: K52.3) are approved for the diagnosis of these diseases.

All the studies using the term IBD in their title and presenting data on the prevalence or incidence (or both) of UC, CD or IBDU (or all) in their text will also be included in this systematic review.

The prevalence of CD, UC or IBDU: The number of patients with $\mathrm{CD}$, UC or IBDU at a given time (point or period of time) in a specific geographical location divided by the total population at risk in that specific location and time per 100000 of the population.

Cumulative incidence of $\mathrm{CD}, \mathrm{UC}$ or IBDU:

Cumulative incidence: Number of new patients with $\mathrm{CD}$, UC or IBDU over a period of at least 1 year in a specific geographical location divided by the total population at risk in that specific location and time per 100000 of the population.

\section{Sampling method and sample size}

Sampling should have been conducted by a random method (simple random sampling, systematic random sampling, stratified random sampling and cluster random sampling or a combination of them) in the preliminary studies that meet the eligibility criteria for this systematic review. The preliminary studies that have used a nonrandom sampling method (quota sampling, convenient sampling, purposive sampling, self-selection sampling and snowball sampling) or public calls will be excluded from this systematic review. The minimum acceptable sample size for the preliminary studies is $30 .^{35}$

\section{Selection process testing (pilot)}

In order to examine the two authors' (MM and ARS) reliability of interpretation of the inclusion criteria and their appropriate classification of the studies, the pilot phase of the selection process will be initially conducted on a sample of papers. This pilot phase will also be used to verify the degree of clarity of the inclusion criteria and to ensure that the criteria are used consistently by both authors.

\section{Search strategy and literature sources \\ Search strategy components}

To achieve the most inclusive search, the search strategy will only be based on the outcome component. To find the equivalent of component, thesaurus systems, including Emtree and $\mathrm{MeSH}$, the free text method, the views of experts and also related articles and abstracts will be used. The other approaches to be used for finding relevant studies include the following.

\section{Electronic database search}

To achieve the study objectives, searches will be carried out in the following electronic databases: PubMed/ MEDLINE, Scopus, WoS (Clarivate Analytics), Embase ( Embase.com) and Google Scholar.

\section{Search in key journals and the reference lists of related studies}

An issue-by-issue manual search will be carried out of two key journals. The journals will be selected based on the analysis of the search outcome of the databases, and a search will be conducted for finding journals presenting the largest pool of sources available on the study subject based on the study inclusion and exclusion criteria. A manual search will also be conducted in the reference lists of the articles selected as the final candidates for quality assessment, and if an article is found in the previous systematic or non-systematic reviews that has been missed out, it will be added to the final articles.

\section{Grey literature}

To find the theses related to the study subject, electronic databases including ProQuest and Scopus will be used in addition to contacting the experts.

Moreover, to obtain relevant conference papers and proceedings, electronic databases will be used in addition 
to the expert information obtained. These references will be searched manually.

\section{Others}

\section{Searching relevant Internet resources}

Since the present study will be conducted on Asian countries, the search will be carried out in the Indian Citation Index, Korean Citation Index, Chinese Citation Index and five other large Chinese biomedical bibliographic databases ${ }^{27}$ and Iran Medex as well as large Asian cohort websites, such as the PERSIAN cohort website in Iran and the Asia-Pacific Crohn's and Colitis Epidemiology Study.

\section{Contacting the experts}

When contacting experts, they will be asked to send any relevant unpublished studies they have as well as any theses by the students working under their supervision. They will also be asked to introduce conferences related to the subject of this systematic review (in addition to the search conducted in the databases).

\section{Date of publication}

All the studies conducted between 1 January 1988 and 30 December 2018, to whose results the researchers have gained access, will be included.

\section{Language of publication}

There will be no language limitations in this systematic review and meta-analysis. The studies that reach the selection stage after screening (based on their title and abstract) and meet the necessary final-stage inclusion criteria and have their full text available and have been written in a language other than English will be translated by Google Translate and rechecked by official translators and then assessed for the final selection.

\section{Constructing the search strategy}

In order to extract the largest number of relevant studies and not miss any related articles, the only component that will be used in the search will be 'outcome (disease)' statements, as shown in table 1 . This syntax is predicted such that it provides the largest possible number of studies (in the electronic database section) by performing the most inclusive search.

This search strategy will be suitable for other electronic databases. Online supplementary appendix 1 of this protocol presents the researchers' complete search syntax for PubMed and Scopus.

All the search stages will be recorded with full details and shall be presented with the final report. All the searches carried out in the various databases will be registered in Endnote.

\section{Study screening and selection}

In order to test the subjects' correct understanding of the inclusion and exclusion criteria during the screening phase, one of the contributors outside the author's team was employed. He was asked to apply the corresponding criteria to two output files with 100 studies (titles and abstracts). This process was performed before the protocol was registered on the PROSPERO.

The search process will be carried out according to the syntaxes related to each electronic database. Then, in the screening stage, two of the authors (ARS and MM) will review the title and abstract of the studies based on a checklist prepared according to the inclusion and exclusion criteria and will find and extract the studies that they identify as related to the study subject. The studies that fail to satisfy even one of these criteria are rejected at this stage. The studies with insufficient data in one or some of the inclusion criteria will be provisionally included in the study, and a final decision will be made about them

\section{Table 1 The search strategy used in PubMed/MEDLINE from 1988 to December 2018}

\begin{tabular}{|c|c|}
\hline Number & Search terms \\
\hline 1 & $\begin{array}{l}\text { ("Idiopathic Proctocolitis"(ti] OR "Ulcerative Colitis"(ti] OR "Colitis Gravis"(ti] OR ("Inflammatory Bowel Disease"(ti] } \\
\text { AND "Ulcerative Colitis Type"(ti))OR"chronic ulcerative colitis"(ti] OR "colitis ulcerative"(ti] OR "colitis ulcerosa"(ti] } \\
\text { OR "colitis ulcerosa chronic"(ti] OR (colitis(ti] AND ulcerative(ti)) OR (Colitis(ti] AND mucosal(ti)) OR (colitis(ti] AND } \\
\text { ulcerous(ti)) OR (Colon(ti] AND "chronic ulceration"(ti)) OR "histiocytic ulcerative colitis"(ti] OR "mucosal colitis"(ti] } \\
\text { OR "ulcerative colorectitis"(ti] OR "ulcerative procto colitis"(ti] OR "ulcerative proctocolitis"(ti] OR "ulcerous } \\
\text { colitis"(ti)) }\end{array}$ \\
\hline 3 & $\begin{array}{l}\text { ("Inflammatory Bowel Disease"(ti] OR ("Bowel Diseases"(ti] AND Inflammatory(ti)) OR "Indeterminate colitis" OR } \\
\text { "undetermined colitis") }\end{array}$ \\
\hline
\end{tabular}


after reviewing their full text in the next stage. Then, in the selection stage, two of the authors (ARS and FE) will review the full text of the studies obtained in the screening stage and determine the final studies, independently, to enter the next stage. Any disagreement in the above two stages will be resolved by consensus, and if the disagreement is not resolved, the opinion of a third expert (KBL) will be used to resolve the case.

\section{Study quality and risk of bias assessment}

To investigate the likelihood of a relationship between the quality of the preliminary studies and their results, the methodological quality of the included studies will be independently assessed by two of the authors (ARS and AK). Any inconsistencies will be resolved by consensus, and if no agreement is reached yet again, the case will be resolved by seeking the views of a third expert (KBL).

The tool to be used is a 10-item tool for assessing the methodological quality of population prevalence studies ${ }^{36}$ and includes the following items:

Items 1 to 4 assess external validity and include the representativeness of the target population, the representativeness of the sample population (sampling frame), random selection and the non-response bias.

Items 5 to 10 assess internal validity and include data collection from the subjects or proxies, acceptable case definition, reliability and validity of the measurement tools, same mode of data collection used for all the subjects, appropriateness of the length of the shortest prevalence period and appropriateness of the numerator(s) and denominator(s).

\section{Data extraction}

Two authors (ARS and MM) will independently complete the data extraction form for all the included studies and then discuss any disagreements to reach a consensus, and if the disagreement is not resolved, the opinion of a third expert will be sought. The following items will be collected and recorded in the data extraction form: study year, publication year, first author's name, journal name, study country, design, setting, target population, sampling method, sample size, total study period, items related to the quality assessment of the study (the score of each item and the total score of the study quality), data related to the prevalence and incidence of IBDs $(\mathrm{CD}$, UC and IBDU, including prevalence, cumulative incidence and incidence rate, based on the measured $95 \%$ CI and $\mathrm{p}$ value) and also, as per the secondary objectives of this study, age and gender groups, geographical region and latitude and the per-capita income of the corresponding country.

In the absence of the required statistical data in the preliminary studies, the authors will attempt to calculate them or communicate with their authors to obtain the data. The study will be eliminated if the author fails to respond to the communications for three times.

\section{Data analysis and synthesis}

The data of each of the included studies will be briefly presented in a table and shall include the first author's name, year of publication, study design and the number and characteristics of the participants. The data related to the incidence and prevalence of $\mathrm{UC}, \mathrm{CD}$ and IBDU will also be presented separately based on the five geographical subgroups (Central Asia, East Asia, Southeastern Asia, Southern Asia and Western Asia).

\section{Statistical analysis}

Pooled analysis

The pooled incidence and prevalence for UC, CD and IBDU will be calculated in this meta-analysis. The combination method will be based on methodological similarities in the included studies by the Fixed Effect Model or the Random Effect Model.

Forest plots will be plotted for all the studies to show the separated and pooled incidence and prevalence and their corresponding 95\% CIs.

The software used in the present study will be Stata V.13.1 (Stata Corp).

\section{Assessment of heterogeneity}

The Q-statistic test and $\mathrm{I}^{2}$ statistic and their corresponding 95\% CIs will be used to assess the statistical heterogeneity of the incidence and prevalence values in the included studies.

The following references will be used as the bases for determining the degree of heterogeneity.

1. Heterogeneity values of $0 \%-40 \%$ will be taken as 'perhaps not important'; 2. Heterogeneity values of $30 \%-60 \%$ as 'moderate heterogeneity'; 3 . Heterogeneity values of $50 \%-90 \%$ as 'substantial heterogeneity'; and 4. Heterogeneity values of $75 \%-100 \%$ as 'considerable heterogeneity'. The level of statistical significance will be set at $\mathrm{p}<0.05$ for the Q-test. ${ }^{37}$

\section{Dose-response relationship evaluation}

According to previous studies, ${ }^{28} 3839$ the latitude of countries and their national income per capita (as a proxy of socioeconomic status) may be somehow associated with the prevalence or incidence of IBDs in them. This review study will calculate these quantitative variables for all the Asian countries that have studies included in this systematic review. The quantitative variables will then be divided into three or more categories. Using the DRMETA command in STATA software, the doseresponse relationship between these two variables and the incidence and prevalence of IBDs will be calculated. The result will show the relationship of the two variables with the diseases under study, and future studies should also consider evaluating mediating variables when examining a disease process.

\section{Temporal trend analysis}

The temporal trends in incidence and prevalence over time will be calculated for the included studies using Joinpoint Regression Program V.4.5.0.1 (Statistical Research 
and Applications Branch, National Cancer Institute). This programme will use the annual prevalence and incidence rates and identify the years in which changes have occurred in the trend of IBDs (join points), and then, using exponentiating beta-coefficients of Poisson regression and subtracting 1, the Annual Percentage Change of these indicators will be calculated between the trend points at a $95 \%$ CI. The programme will also calculate the Average Annual Percentage Change (AAPC) for the entire study period. ${ }^{4041}$

\section{Subgroup analysis}

In the present study, in addition to geographical subgroups (Central Asia, Eastern Asia, Southeastern Asia, Southern Asia and Western Asia), the studies will be divided into different subgroups and analysed based on the geographical latitude of their corresponding countries, the percapita income of their corresponding countries, age group, gender, method of sampling, and so forth.

\section{Assessment of publication bias}

The first strategy to deal with publication bias is performing the most inclusive search in the search stage of the study.

Also, funnel plots will be used to assess potential reporting bias and non-significant study effect.

Begg's test and Egger's test will also be performed, and significant results $(p>0.1)$ shall suggest a publication bias, in which case the 'trim and fill' method will be used.

\section{Sensitivity analysis}

A sensitivity analysis will also be performed in this study to assess methodological quality, design limitations, data analysis considerations, sample size and effect of missing data.

The sensitivity analysis will be based on the one-out remove method, in which the other studies are pooled and compared with each other with one of the studies left out each time.

\section{Quality analysis}

For the quality analysis, the relationship between the methodological quality index of the preliminary studies and their results (prevalence or incidence values) will be assessed. If there are significant differences between the results of the high-quality methodological studies and the results of the poor-quality methodological studies, a combination of the studies with a minimum acceptable quality will be used as a valid and reliable estimate of the combination of these studies.

\section{Missing data}

In the case of missing data in the final included studies, attempts will be made to access the authors' contact data and complete the missing data by corresponding with them. The lack of access to sufficient data (after sending three emails) shall necessarily mean the elimination of that study from the data synthesis process.
Patient and public involvement

No patients will be involved in this study.

\section{DISCUSSION}

This systematic review and meta-analysis study will estimate the pooled incidence and prevalence of UC, CD and IBDU in the Asian continent. It will also provide evidence of the causes for the high variation in the reported incidence and prevalence of these diseases in Asian countries. Since this study will use comprehensive and meticulous methods in all the steps of the systematic review and meta-analysis, the information obtained will be completely reliable.

Some of the most important limitations of the future study will be the high level of heterogeneity in prevalence studies because of the dependence of these studies on time and place, the lack of strong population-based studies in most countries of the Asian continent and probable methodological bias in including the primary studies.

Given the increasing pattern of these diseases in developing countries, the information gathered from this study can be widely used by physicians, health policymakers and custodians to allocate funds to the prevention and treatment of these major diseases in Asian countries.

Acknowledgements Hereby, the authors wish to express our gratitude to Professor Yunes Ghasemi, the Vice Chancellor of Research at Shiraz University of Medical Sciences for his specific support from systematic review and metaanalysis projects.

Contributors ARS is the guarantor of this study. All the authors contributed to the conception and design of the protocol as follows. ARS worked on the topic refinement, formulation of research question, review design, study selection forms, data extraction sheets and plan of analysis and wrote the protocol; ARS also designed the search strategy under the supervision of $A K, K B L, M M$ and $F E$. KBL, MM and AK contributed to the topic refinement, formulation of research question, review design and plan of analysis and gave critical feedback on the intellectual content of the draft protocol. KBL, AK, FE and MM reviewed the manuscript for feedback. MM will review the articles and take care of the data extraction step along with ARS. AK, MM and FE will take care of database management and carry out the literature search/handle the bibliography. As the senior author, KBL supervised the preparation of the study protocol and addressed the reviewers' comments. All the authors read and approved the final manuscript.

Funding This research received no specific grant from any funding agency in the public, commercial or not-for-profit sectors.

Competing interests None declared.

Patient consent for publication Not required.

Provenance and peer review Not commissioned; externally peer reviewed.

Open access This is an open access article distributed in accordance with the Creative Commons Attribution Non Commercial (CC BY-NC 4.0) license, which permits others to distribute, remix, adapt, build upon this work non-commercially, and license their derivative works on different terms, provided the original work is properly cited, appropriate credit is given, any changes made indicated, and the use is non-commercial. See: http://creativecommons.org/licenses/by-nc/4.0/.

\section{REFERENCES}

1 Kaplan GG. The global burden of IBD: from 2015 to 2025. Nat Rev Gastroenterol Hepatol 2015;12:720-7.

2 Abraham C, Cho JH. Inflammatory bowel disease. N Engl J Med 2009;361:2066-78. 
3 Orholm M, Binder V, Sørensen TI, et al. Concordance of inflammatory bowel disease among Danish twins. Results of a nationwide study. Scand J Gastroenterol 2000;35:1075-81.

4 Hampe J, Heymann K, Krawczak M, et al. Association of inflammatory bowel disease with indicators for childhood antigen and infection exposure. Int J Colorectal Dis 2003;18:413-7.

5 Cruickshank SM, McVay LD, Baumgart DC. Colonic epithelial cell mediated suppression of CD4 T cell activation. Gut 2004;53:678-84.

6 Sands BE. From symptom to diagnosis: clinical distinctions among various forms of intestinal inflammation. Gastroenterology 2004;126:1518-32.10.1053/j.gastro.2004.02.072

7 Lennard-Jones JE. Classification of inflammatory bowel disease. Scand J Gastroenterol 1989;24:2-6. discussion 16-9.

8 Calkins BM, Lilienfeld AM, Garland CF, et al. Trends in incidence rates of ulcerative colitis and Crohn's disease. Dig Dis Sci 1984;29:913-20.

9 Myren J, Bouchier IA, Watkinson G, et al. The OMGE multinational inflammatory bowel disease survey 1976-1986. A further report on 3175 cases. Scand J Gastroenterol Suppl 1988;144:11-19.

10 Shivananda S, Hordijk ML, Ten Kate FJW, et al. Differential diagnosis of inflammatory bowel disease: a comparison of various diagnostic classifications. Scand J Gastroenterol 1991;26:167-73.

11 Guindi M, Riddell R. Indeterminate colitis. J Clin Pathol 2004;57:1233-44.

12 Ouyang Q, Tandon R, Goh KL, et al. Management consensus of inflammatory bowel disease for the Asia?Pacific region. $J$ Gastroenterol Hepatol 2006;21:1772-82.

13 Molodecky NA, Soon IS, Rabi DM, et al. Increasing incidence and prevalence of the inflammatory bowel diseases with time, based on systematic review. Gastroenterology 2012;142:46-54.

14 Cosnes J, Gower-Rousseau C, Seksik P, et al. Epidemiology and natural history of inflammatory bowel diseases. Gastroenterology 2011:140:1785-94.

15 Kappelman MD, Moore KR, Allen JK, et al. Recent Trends in the Prevalence of Crohn's Disease and Ulcerative Colitis in a Commercially Insured US Population. Dig Dis Sci 2013;58:519-25.

16 Rocchi A, Benchimol El, Bernstein CN, et al. Inflammatory bowel disease: a Canadian burden of illness review. Can J Gastroenterol 2012;26:811-7.10.1155/2012/984575

17 Burisch J, Jess T, Martinato M, et al. The burden of inflammatory bowel disease in Europe. J Crohns Colitis 2013;7:322-37.

18 Thia KT, Loftus, Jr EV, Sandborn WJ, et al. An update on the epidemiology of inflammatory bowel disease in Asia. Am J Gastroenterol 2008;103:3167-82.

19 SC N, Tang W, Ching JY, et al. Incidence and phenotype of inflammatory bowel disease based on results from the AsiaPacific Crohn's and colitis epidemiology study. Gastroenterology 2013;145:158-65.

20 Zhao J, SC N, Lei Y, et al. First prospective, population-based inflammatory bowel disease incidence study in mainland of China: the emergence of "western" disease. Inflammatory bowel diseases 2013;19:1839-45.

21 Morita N, Toki S, Hirohashi T, et al. Incidence and prevalence of inflammatory bowel disease in Japan: nationwide epidemiological survey during the year 1991. J Gastroenterol 1995;30:1-4.

22 Asakura $\mathrm{K}$, Nishiwaki $\mathrm{Y}$, Inoue N, et al. Prevalence of ulcerative colitis and Crohn's disease in Japan. J Gastroenterol 2009;44:659-65.
23 Yang S-K, Yun S, Kim J-H, et al. Epidemiology of inflammatory bowel disease in the Songpa-Kangdong district, Seoul, Korea, 1986-2005: a KASID study. Inflamm Bowel Dis 2008;14:542-9.10.1002/ibd.20310

24 Cole EB, Shah Y, McLean LP, et al. Frequency of surveillance and impact of surveillance Colonoscopies in patients with ulcerative colitis who developed colorectal cancer. Clin Colorectal Cancer 2018;17:e289-92.

25 SC N, Shi HY, Hamidi N, et al. Worldwide incidence and prevalence of inflammatory bowel disease in the 21st century: a systematic review of population-based studies. The Lancet 2017;390:2769-78.

26 Prideaux L, Kamm MA, De Cruz PP, et al. Inflammatory bowe disease in Asia: a systematic review. J Gastroenterol Hepatol 2012;27:1266-80.

27 Xia J, Wright J, Adams CE. Five large Chinese biomedical bibliographic databases: accessibility and coverage. Health Info Libr $J$ 2008;25:55-61.

28 Bernstein CN, Wajda A, Svenson LW, et al. The epidemiology of inflammatory bowel disease in Canada: a population-based study. Am J Gastroenterol 2006;101:1559-68.

29 Kaplan GG, Ng SC. Understanding and preventing the global increase of inflammatory bowel disease. Gastroenterology 2017; 152:313-21.

30 Nations U, 2018. Available: https://unstats.un.org/unsd/ methodology/m49

31 Centre for Reviews \& Dissemination. Systematic reviews: CRD's guidance for undertaking reviews in healthcare. New York: University of York NHS Centre for Reviews \& Dissemination, 2009.

32 Stroup DF, Berlin JA, Morton SC, et al. Meta-Analysis of observational studies in epidemiology: a proposal for reporting. Jama 2000;283:2008-12.

33 Moher D, Liberati A, Tetzlaff J, et al. Preferred reporting items for systematic reviews and meta-analyses: the PRISMA statement. PLoS Med 2009;6:e1000097.

34 Satsangi J, Silverberg M, Vermeire S. The Montreal classification of inflammatory bowel disease: controversies, consensus, and implications. Gut 2006;55:749-53.

35 Chen H-Y, Shen D-T, Ji D-Z, et al. Prevalence and burden of hepatitis $D$ virus infection in the global population: a systematic review and meta-analysis. Gut 2019;68:512-21.

36 Hoy D, Brooks $\mathrm{P}$, Woolf $\mathrm{A}$, et al. Assessing risk of bias in prevalence studies: modification of an existing tool and evidence of interrater agreement. J Clin Epidemiol 2012;65:934-9.

37 Higgins JPT, Thompson SG, Deeks JJ. Measuring inconsistency in meta-analyses. BMJ 2003;327:557-60.

38 SC N, Kaplan G, Banerjee R, et al. 78 incidence and phenotype of inflammatory bowel disease from 13 countries in Asia-Pacific: results from the Asia-Pacific Crohn's and colitis epidemiologic study 2011 2013. Gastroenterology 2016;150:S21.

39 Ko Y, Kariyawasam V, Karnib M, et al. Inflammatory bowel disease environmental risk factors: a population-based case-control study of middle Eastern migration to Australia. Clin Gastroenterol Hepatol 2015;13:1453-63.

40 Dragomirescu I, Llorca J, Gómez-Acebo I, et al. A join point regression analysis of trends in mortality due to osteoporosis in Spain. Sci Rep 2019;9:4264.

41 Jones G-R, Lyons M, Plevris N, et al. Ibd prevalence in Lothian, Scotland, derived by capture-recapture methodology. Gut 2019;68:1953-60. 